\title{
Dust production in supernovae: the case of Kepler's SNR
}

\author{
E. M. Reynoso ${ }^{1} \dagger$, H. L. Gomez ${ }^{2}$ and L. Dunne ${ }^{3}$ \\ ${ }^{1}$ IAFE (CONICET-UBA) and Physics Dept. (FCEyN, UBA), Buenos Aires, Argentina \\ ${ }^{2}$ School of Physics and Astronomy, Cardiff University, The Parade, Wales, UK \\ ${ }^{3}$ School of Physics and Astronomy, University of Nottingham, UK
}

\begin{abstract}
We compare the submillimetre (submm) emission with the $\mathrm{H}$ I and $\mathrm{CO}$ distribution towards Kepler's supernova remnant (SNR), and conclude that 0.1 to $1.2 \mathrm{M}_{\odot}$ of dust originates from Kepler. Such rates are sufficient to explain the origin of dust in high redshift galaxies.
\end{abstract}

Keywords. dust, supernova remnants, individual: Kepler's SNR, submillimetre

\section{Results and conclusions}

The role of supernovae as significant sources of dust is still unresolved. To explore this subject, we analyze the submm emission towards Kepler's SNR - Morgan et al. 2003 - in connection with the 3-dimensional picture of the interstellar medium (ISM) around this source as inferred from $\mathrm{H} \mathrm{I}$ and $\mathrm{CO}$ line data, with the aim of differentiating the amount of dust corresponding to the ISM or to the remnant. To ensure that the submm emission structure is not affected by the observing technique employed, simulations of chopping over different possible emission distributions were carried out.

We find a ring-like structure of submm emission coincident with the remnant's shell, which cannot be explained by a synchrotron source, chopping on-off nearby interstellar structures, swept-up interstellar material, nor by line-of-sight contamination. Indeed, there is no correlation between the submm peaks and the $\mathrm{H}$ I or $\mathrm{CO}$ emission, while the molecular gas is found to be diffuse and optically thin and has a negligible contribution to the submm emission towards the SNR. After subtracting a synchrotron component using a spectral index map, we estimate a dust mass of 0.1 or $1.2 \mathrm{M}_{\odot}$ depending on the absorption coefficient (Gomez et al. 2009). This result agrees with the range of estimates for Cas A (from IR and submm data: Krause et al. 2004, Rho et al. 2008, Dunne et al. 2009) and implies that supernovae can still be considered as significant sources of dust.

\section{Acknowledgements}

E. M. R. is partially supported by grants PIP-CONICET 114-200801-00428 and UBACyT X482. H.L.G. would like to acknowledge the support of LCOGT.

\section{References}

Dunne L. et al., 2009, MNRAS, 394, 1307

Gomez, H. L., Dunne, L., Ivison, R. J., Reynoso, E. M., Thompson, M. A., Sibthorpe, B., Eales,

S. A., DeLaney, T. M., Maddox, S., \& Isaak, K. 2009, MNRAS 397, 1621

Krause O., et al., 2004, Nature, 432, 596

Morgan H. L., Dunne L., Eales S., Ivison R. J., \& Edmunds M. G., 2003, ApJ, 597, L33

Rho J., et al., 2008, ApJ, 673, 271

$\dagger$ Member of the Carrera del Investigador Cient’ıfico, CONICET, Argentina. 